\title{
Field-Validated Communication Systems for Smart Microgrid Energy Management in a Rural Microgrid Cluster
}

\author{
Hiranmay Samanta ${ }^{1}$, Abhijit Das ${ }^{1}$, Indrajt Bose ${ }^{1}$, Joydip Jana ${ }^{1}$, Ankur Bhattacharjee ${ }^{2, *}$, \\ Konika Das Bhattacharya ${ }^{3}$, Samarjit Sengupta ${ }^{1}$ and Hiranmay Saha ${ }^{1}$ \\ 1 School of Advanced Materials, Green Energy and Sensor Systems, IIEST, Shibpur, Howrah 711103, India; \\ hiranmaysamanta@gmail.com (H.S.); abhijitds7@gmail.com (A.D.); indrajitbose98@gmail.com (I.B.); \\ joydipjana02@gmail.com (J.J.); samarsgp@gmail.com (S.S.); sahahiran@gmail.com (H.S.) \\ 2 Department of Electrical and Electronics Engineering, BITS-Pilani, Hyderabad Campus, \\ Hyderabad 500001, India \\ 3 Department of Electrical Engineering, IIEST, Shibpur, Howrah 711103, India; kdb@ee.iiests.ac.in \\ * Correspondence: a.bhattacharjee@hyderabad.bits-pilani.ac.in; Tel.: +91-9749397160
}

check for updates

Citation: Samanta, H.; Das, A.; Bose,

I.; Jana, J.; Bhattacharjee, A.;

Bhattacharya, K.D.; Sengupta, S.;

Saha, H. Field-Validated

Communication Systems for Smart Microgrid Energy Management in a Rural Microgrid Cluster. Energies 2021, 14, 6329. https://doi.org/ 10.3390/en14196329

Academic Editor: Abu-Siada Ahmed

Received: 15 July 2021

Accepted: 2 September 2021

Published: 4 October 2021

Publisher's Note: MDPI stays neutral with regard to jurisdictional claims in published maps and institutional affiliations.

Copyright: (C) 2021 by the authors. Licensee MDPI, Basel, Switzerland. This article is an open access article distributed under the terms and conditions of the Creative Commons Attribution (CC BY) license (https:/ / creativecommons.org/licenses/by/ $4.0 /)$.

\begin{abstract}
This paper demonstrates a smart energy management scheme for solar photovoltaicbiomass integrated grid-interactive microgrid cluster system. Three interconnected microgrids were chosen as a cluster of microgrids for validation of the proposed community energy management scheme. In this work, a Global System for Mobile (GSM)-based bidirectional communication technique was adopted for real-time coordination among the renewable energy sources and loads. To realize the common phenomenon of local grid outage in rural distribution networks, a practical case study is designed in this work. The optimized scheduling of the energy sources and loadsof different microgrids and the distribution grid were implemented to ensure zero loss of power supply probability (LPSP) for dynamic load profiles. The laboratory-scale prototype of the proposed microgrid clustering was first developed in this work by establishing real-time communication among multiple energy sources and loads through different energymeters located at different places inside the academic campus. The field validation was performed with a microgrid cluster consisting of $45 \mathrm{~kW}_{\mathrm{P}}$ solar photovoltaic, $50 \mathrm{kVA}$ biogas plant, community loads in a village. The developed smart energy management solution is a generalized one and applicable to satisfy scalable community energy demands as well.
\end{abstract}

Keywords: Internet of Things (IoT); microgrid cluster; renewable energy; smart energy management

\section{Introduction}

The rapid growth of demand for electric energy has become the primary requirement of today's world, especially in developing countries where the growth rate of electric power demand is much higher. The rural parts of all countries seriously require a sustainable source of energy to meet their needs. By contrast, the energy requirement of the urban areas is mainly for commercial purposes, which in turn is expected to contribute to the nation's gross domestic product (GDP). The effectiveness of implementing a microgrid consisting of distributed energy resources (DER) and loads generally built to cater to the local energy demand, with the ability to operate in both grid-connected and islanded modes, needs to be studied. This type of model may be becoming necessary for the rural regions, especially for developing countries such as India.

The sources of electric energy of a microgrid are classified into three categories, primary sources, alternative sources, and the energy storage system. The selection of energy sources of a microgrid depends on its availability. A microgrid situated in a hilly region has a micro-hydro turbine or a solar plant as its primary source [1].

In microgrids, besides the interconnection among the renewable energy sources (RES), real-time scheduling is also essential at both the generation and load end [2,3]. A micro- 
grid controller schedules the microgrid sources (MS) and load controller (LC) to achieve optimal energy management. An appropriate combination of multiple RES operating through a distinct network for specified load patterns forming a smart microgrid can satisfy demand response management(DRM) [4-6]. The control technique for enhancing the stable operation of distributed generation units within a microgrid was demonstrated in various papers [7]. In order to achieve optimized energy management [8], one of the core performance requirements of a smart microgrid is two-way communication between the energy sources and the microgrid controller. A microgrid controller schedules the microgrid sources (MS) and load controller (LC) to ensure zero LPSP. This entails that the load is always satisfied by the microgrid sources $[9,10]$. In order to achieve zero LPSP, it is essential to manage and control the microgrid sources and load controllers.

Some research papers have discussed rural microgrid models for residential and agricultural systems, where precise energy scheduling has been carried out based on load classification and forecasting [11]. Different algorithms are used to predict the short-term power consumption by two types of load. Cloud energy storage (CES) is used to optimize energy storage cost and energy utilization [12]. The energy management systems (EMS) are also working for microgrid operations, which are based on different models of the control strategy. The EMS also focuses on optimizing the cost and energy utilization of a microgrid [13].

The microgrid cluster helps to exchange power generation within a group of microgrids. However, the microgrids should be situated within a certain distance. A microgrid cluster can be connected to the distribution grid or operate in islanded mode. The reasons to implement a microgrid cluster are to mitigate the energy outage of a particular location and ensure $24 \times 7$ energy availability. If an energy deficit happens in a microgrid, a string of microgrids or microgrid clusters can recover the energy deficit through a commonly connected AC bus. The different frameworks of the architecture of the microgrid cluster are shown in Figure 1. Connections in a microgrid cluster may be in series, parallel, or both combinations [14-16].

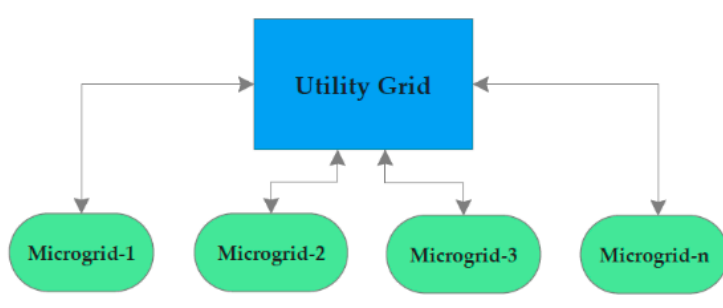

Parallelly connected microgrid, connected with utility grid
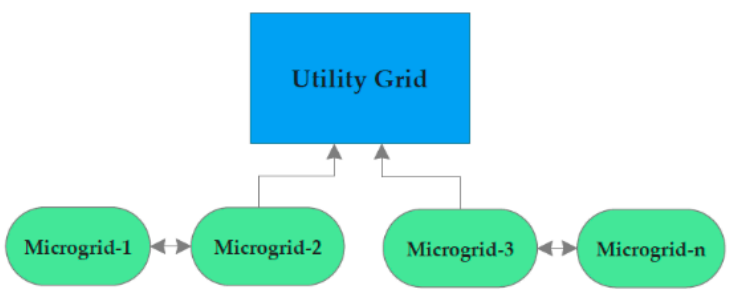

Serially interconnected microgrid, connected with utility grid

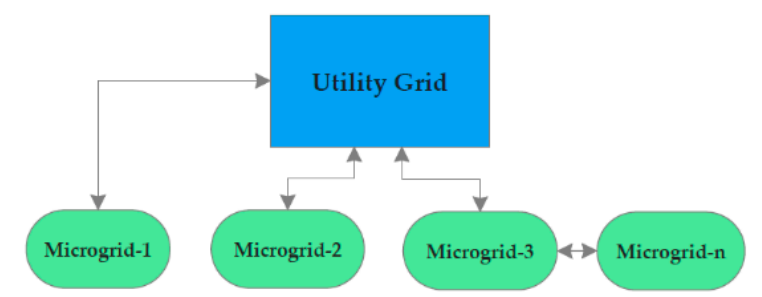

Parallel-series connection of microgrids connected with utility grid

Figure 1. Power router architecture of microgrid cluster.

In recent days, internet-based low-cost supervisory control and data acquisition (SCADA) are used to communicate and control a microgrid locally or remotely $[17,18]$. Over the past few years, there has been a growing awareness regarding the concept of the Internet of Things (IoT), which involves connecting to the internet various objects surrounding us in everyday life $[19,20]$. The primary basis of this concept is closely related to networking for smart cities, and this approach is increasingly enhancing the quality of life by contributing to streamlining resource consumption and protecting the 
environment [21,22]. In this era of IoT and cloud-based storage systems, it is easy to implement internet-based control and management system for a microgrid or cluster of microgrids. The major facility of using IoT-based communication in a microgrid system is its open-source compatibility. The remote monitoring and control of microgrids become easier to implement by using this platform. Different papers have established the microgrid management system using the IoT-based platform [19].

The communication medium of the Internet of Things (IoT)-based system depends on their requirements, which can vary based on the data rate, communication range, power consumption, line of sight issue, and so on. For indoor applicable small range low data rate required to communicate with each other, Bluetooth communication can be used, whereas, in the long-range outdoor application and low data rate communications system, Zig-bee or Long-Range (LoRa) can be used. To implement a smart microgrid system in rural areas, the line of sight may be a barrier for uninterrupted communication of the data. The security issue with the communication medium can be a barrier too. A comparative analysis is shown in Table 1 for the selection of different communication mediums [23].

Table 1. Comparative analysis among the communication platforms for IoT-based microgrid cluster and management applications [23].

\begin{tabular}{|c|c|c|c|c|c|c|}
\hline Specification & Bluetooth & Zig-Bee & LoRa & $\begin{array}{l}\text { WiMAX } \\
\text { (3G/4G) }\end{array}$ & GSM/GPRS & $\begin{array}{c}\text { Internet over } \\
\text { Wi-Fi }\end{array}$ \\
\hline $\begin{array}{l}\text { Distance } \\
\text { coverage }\end{array}$ & $100 \mathrm{~m}-400 \mathrm{~m}$ & $\begin{array}{l}\text { Up to } 3.2 \mathrm{~km} \\
\text { Outdoor RF } \\
\text { line-of-sight } \\
\text { range }\end{array}$ & Up to $15 \mathrm{~km}$ & Up to $10 \mathrm{~km}$ & $\mathrm{Up}$ to $50 \mathrm{~km}$ & $300 \mathrm{~m}$ \\
\hline $\begin{array}{l}\text { Data transfer } \\
\text { rate }\end{array}$ & Up to $2 \mathrm{Mbps}$ & Up to $256 \mathrm{Kbps}$ & Up to $37.5 \mathrm{Kbps}$ & Up to $100 \mathrm{Mbps}$ & $512 \mathrm{Kbps}$ & Up to $54 \mathrm{Mbps}$ \\
\hline $\begin{array}{c}\text { Power } \\
\text { consumption } \\
\text { (W) }\end{array}$ & 100 mW Max & $3.7 \mathrm{~W}$ & $4.2 \mathrm{~W}$ & $72 \mathrm{~W}$ & $1 \mathrm{~W}-2 \mathrm{~W}$ & $4.9 \mathrm{~W}$ \\
\hline $\begin{array}{l}\text { Multi point } \\
\text { connection }\end{array}$ & No & Yes & Yes & Yes & Yes & Yes \\
\hline Security & No & Yes & Yes & Yes & Yes (Encrypted) & Yes \\
\hline $\begin{array}{l}\text { Expansion } \\
\text { capability }\end{array}$ & Yes & Yes & Yes & No & Yes & Yes \\
\hline
\end{tabular}

Having a high data communication rate, data security, and no line of sight issue, a GSM-based communication system can be used to establish proper communication between two or more microgrids in the rural application, which helps to achieve zero LPSP. Various energy meters are associated with energy sources and loads in the different microgrid systems. Each energy meter is connected with a separate communication system having a standard industrial protocol. Different switches and communication protocols are used to control and communicate the DERs in the microgrids. The management and scheduling of sources and loads of microgrids are essential for stable and continuous operation. For a rural microgrid, where there is more uncertainty regarding centralized electricity, clustering of microgrids can be taken into consideration.

For rural microgrid clustering, the communication between the microgrids is essential for balancing the local load demand using different microgrid sources. However, in the rural region, the network connectivity is very uncertain. The other communication mediums can be used judiciously, depending on the requirements. In the case of establishing communication between the two or more rural microgrids, communication redundancy and a stable communication medium are essential. Table 1 shows the comparison between different communications mediums. In this case, we are using GSM as the primary communication medium for its network coverage, range, security, and stable mode operation.

Here, the primary objective is to satisfy community-scale energy management by establishing smart communication for clustered microgrids under the rural scenario. How- 
ever, the power purchase and/or commercial aspect of power distribution in microgrid has not been taken into account. Therefore, the privacy issue of the communication network of microgrid was not considered in this work. However, it is required to be considered in the case of commercial analysis of power distribution in microgrids.

A practical field application of the proposed smart energy management technique is shown in this work by considering a cluster of three microgrids comprising a total capacity of $45 \mathrm{kWp}$ distributed solar photovoltaic system (PV) and $50 \mathrm{kVA}$ of a distributed biogas plant. The local distribution grid was considered a rural grid. Throughout the day, dynamic load profiles of the three sub-areas were considered for experimental validation.

Such a type of GSM-based smart communication scheme for clustered microgrid energy management ensuring zero loss of power supply probability (LPSP) has not been reported so far, to the best of our knowledge. Therefore, the field validation of the proposed work is shown to justify its novelty.

The rest of the paper is organized as follows: Section 2 includes the operational architecture of microgrid and clusters of microgrid, different communication systems used for prototype microgrid cluster control, and management on IoT-based platform; Section 3 describes a practical example of a rural microgrid application using a novel cluster control method; Section 4 describes the result; and finally, Section 5 includes the conclusion of this paper.

\section{Architecture of the Microgrid}

A microgrid allows different RES within it and can be operated in the grid-connected distribution as well as in islanded mode. A microgrid strengthens the distribution grid resilience in a rural area, where it is weak in nature; it is essential for a remote location where the national distribution grid is not present or is weak, and microgrids can support the critical loads effectively.

The microgrid consists of distributed energy resources (DER) and connected loads within its distribution network. An intelligent centralized controller is used to manage the generation and demand of a microgrid. A microgrid system can be connected to the distribution grid at any point of common coupling (PCC). Figure 2 shows the basic architectural diagram of a microgrid. When a microgrid is in grid-connected mode, it can take or give the power partially from the distribution grid if an energy deficit or surplus happens. In addition, in Islanded mode, it can manage the required and generated power within it. A microgrid is capable of driving local loads in an uninterrupted way in the absence of a grid.

Such systems' technical and economic aspects forecast a steady growth of microgrids that can sustain the availability of power in remote regions in any part of the globe.

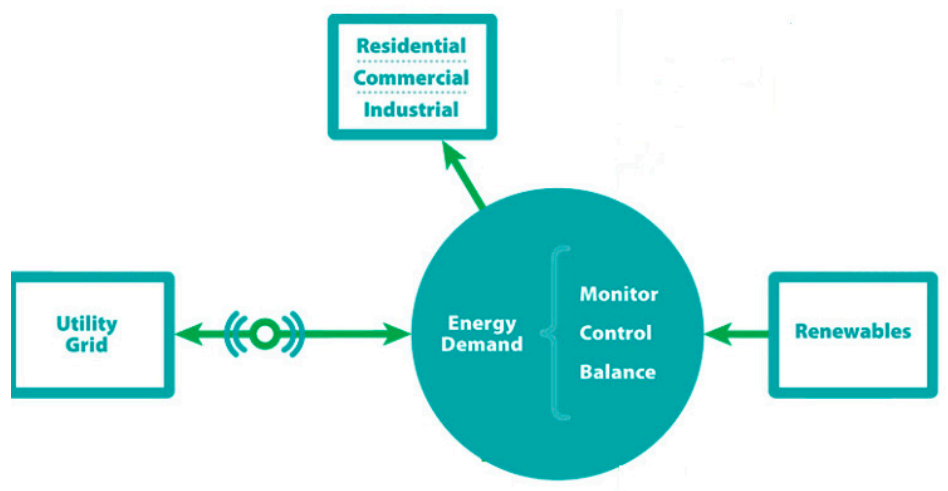

Figure 2. Basic microgrid architecture.

\subsection{Clustering of Microgrid}

Two or more microgrids are connected to form a microgrid cluster [24]. The microgrid cluster aims to satisfy the community load from different DERs and has less dependency on 
the distribution grid. The pattern of the connections of microgrids can be different, as shown in Figure 1. Bidirectional communication is used to optimize the operation of a microgrid cluster or manage the microgrid sources and the loads [14-16]. The communication and control schemes of the microgrid cluster are shown in Figure 3.

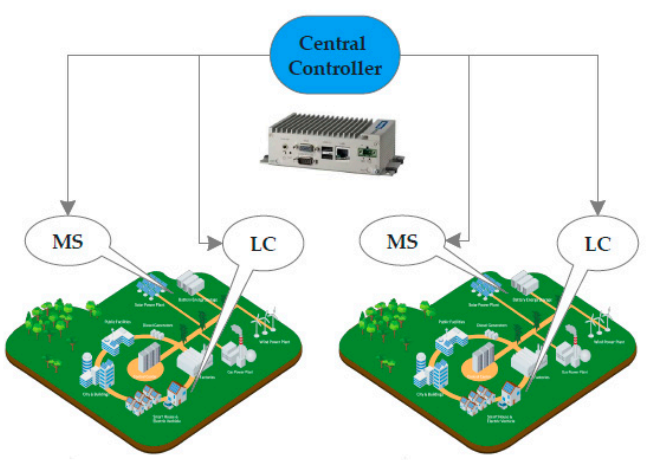

(a) Centralized microgrid cluster scheme

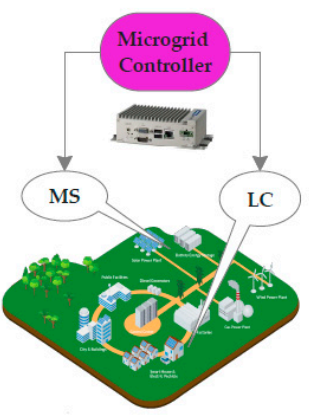

(b) Decentralized microgrid cluster scheme
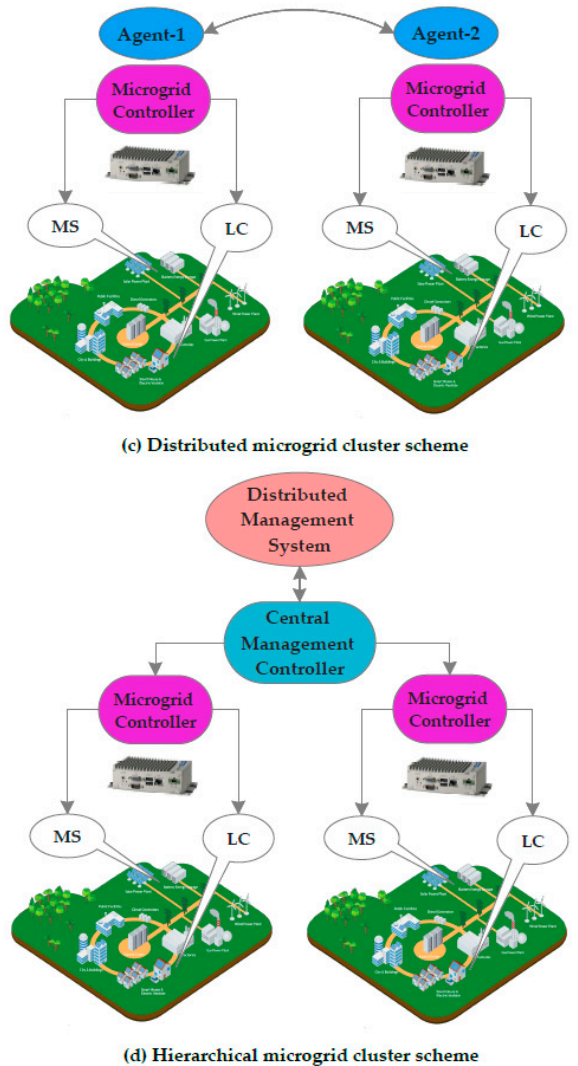

Figure 3. Control scheme of different types of microgrid cluster. (a) Centralized; (b) decentralized; (c) distributed; and (d) hierarchical.

Ina centralized control mode of operation, a single central controller is responsible for its control actions in every microgrid source (MS) and load controller (LC), as shown in Figure 3a. In this approach, the controller communicates with each microgrid component $[14,15]$. The major drawback of this method lies in the centralized control system; any single point fault can destabilize the whole microgrid cluster. In a decentralized control mode of operation, the individual controller is responsible for its operation and control, as shown in Figure $3 \mathrm{~b}$. This approach provides an independent control with a data-sharing capability with any microgrid cluster. However, optimal operation can be affected in the absence of a communication link with the cluster unit [14,15].

In distributed control mode of a microgrid operation, different microgrid controllers or agents coordinate for an optimal operation, as shown in Figure 3c. In this topology, two-way communication is required to allow data sharing between separate microgrid controllers and agents. As all the data are shared with each other, security is a concern for this type of system. In the hierarchical control mode of operation, the system is divided into multiple control layers with distinct control tasks and goals, as represented in Figure 3d. For instance, the hierarchical control of a microgrid cluster may operate with multiple microgrid controllers and loads in the first control layer [14,15]. A central management controller (CMC) will take care of all microgrid controllers in the cluster $[25,26]$. The CMC will connect to the distributed management system (DMS) in the third control layer.

Microgrids can operate in an interconnected microgrid network to share the generation and demand, which is called the clustering of the microgrid [27]. The benefits of this 
kind of system can be seen in both grid-connected and the islanded mode of operations. All microgrids participate in a network where they can share and fulfill their energy requirements [15]. However, this kind of coordination requires a stable operation of each microgrid and reliable communication between them [28]. The essential things for a cluster of microgrids are the communication between them. When the microgrids share their sources to contribute their energy demand in the islanded mode of operation, it is essential to schedule their sources to avoid voltage swelling or sagging in the bus. Here, the communication plays a significant role in the operation of sources to prevent such situations. Therefore, a stable mode of communication can make the microgrid cluster stable.

In the rural areas, the availability of biogas is much more than in the urban area, along with other renewable sources such as solar PV and wind energy. This means that more energy can be available from the biogas when the energy demand is high. A microgrid totally depends on its natural energy resources, but it is always difficult to establish a microgrid in a proper location, where the energy demand is high. However, using the clustering mode of microgrid operation, it is easy to ensure the energy demand even in a highly populated rural area. A practical problem is discussed in this paper, where three microgrids are located in three different locations. The line flow constraints and capacity limits are essential issues for a microgrid cluster. However, in the field case study shown in this paper, the distance between the microgrids is less than $1 \mathrm{~km}$, and the capacity of each microgrid is in the range of a few $\mathrm{kW}$; hence, the said constraints were not considered here.

The prototype of the microgrid cluster established at the IIEST, Shibpur campus, can be applied for those villages where different microgrids could meet their own local energy demands [29]. Figure 4 shows four microgrids clustered to meet the energy demand with zero LPSP. In this cluster, microgrid 1 and microgrid 2 have the RES (solar PV andbiomass) connected, whereas microgrid 3 and microgrid 4 comprise of local grids and loads. All microgrids (MG-1, MG-2, MG-3, and MG-4) are connected to a local AC distribution grid. The field validation of the proposed microgrid cluster energy management is performed by three interconnected microgrids under therural scenario. These microgrids contain bio-solar energy sources and can communicate in real time by exchanging power when required, using a GSM-based smart communication platform, thus forming the clustered microgrid.
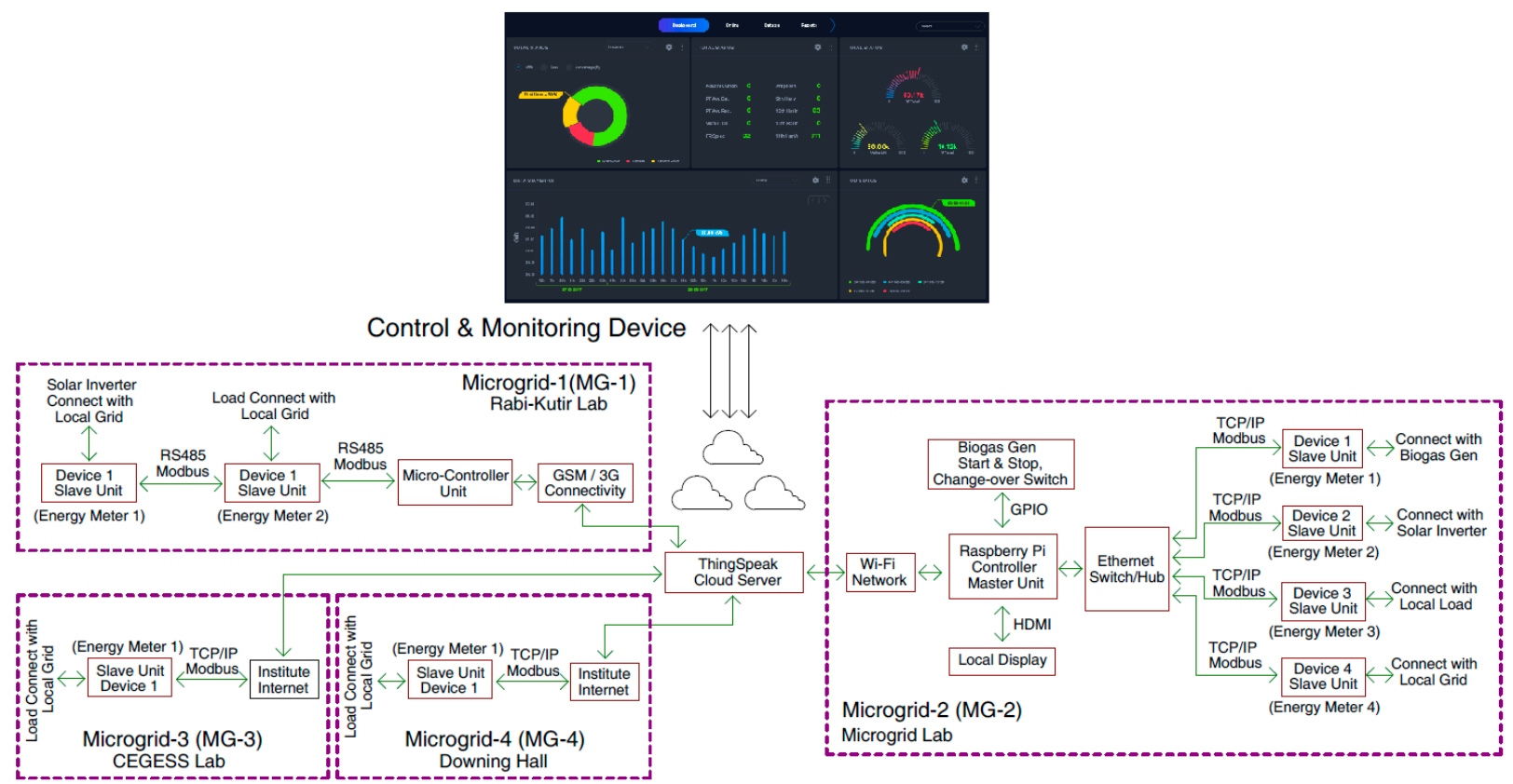

Figure 4. Schematic of the prototype microgrid cluster usinga different communication platform. 


\subsection{GSM-Based Microgrid}

In Figure 4, four subsystems consisting of energy sources and loads are connected to build a cluster of microgrids. The control and management of each microgrid are individual and different. All microgrids are connected to the local grid in parallel, and the communication scheme is hierarchical. All the microgrids are connected to a central monitoring and control device via an IoT platform. Microgrid-1 connects to energy meters to measure the energy consumed by the load and power generated by the energy sources. The power and energy data are fetched by the controller unit, and then the information is communicated to the central management controller unit (CMC).

The energy source (solar PV) and loads of microgrid-1 are connected via energy meters, as shown in Figure 5. The data are collected by the microcontroller unit using industrystandard RS485 Modbus communication. The peripheral modules such as a real-time clock (RTC), SD memory cards, and relays are connected with the microcontroller unit to serve the real-time control. The relays, as shown in Figure 5, are used to schedule the local loads of microgrid-1. Finally, a GSM-based modem is used to communicate between the microgrid controller unit and the Thing Speak server. The MATLAB Thing Speak is a graphical user interface (GUI)-based middleware used to present the data graphically [18]. The open-source application Thing Speak can connect cloud storage or local Message Queuing Telemetry Transport (MQTT) broker to analyze mass data almost in realtime [17].

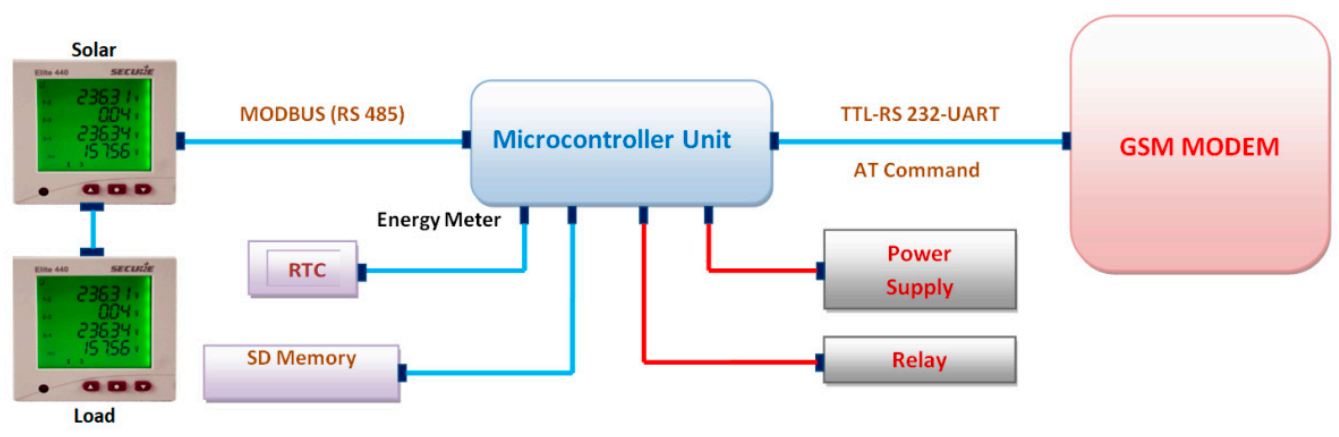

Figure 5. Schematic diagrams of GSM and microcontroller-based microgrid (Microgrid-1).

\subsection{Wi-Fi-Based Microgrid}

In microgrid-2, four energy meters are connected with two energy sources (solar PV and biogas), loads, and the distribution grid, as shown in Figure 6. All energy meters are connected to the controller unit via industry-standard Modbus over TCP/IP protocol. Another microcontroller unit is used to start or stop the biogas generator. This microcontroller unit is also communicating with the Raspberry unit for energy management purposes. That is to say, the raspberry pi controller is used as a microgrid controller, which is further communicated and control by the CMC to form the hierarchical scheme of microgrid clustering. Here, the Raspberry Pi is connected to the Thing Speak server via a Wi-Fi connection. Microgrid-2 has two sources: a local load and a combined distribution grid.

For both the microgrids 1 and 2, the purpose of the communication medium (GSM/Wi$\mathrm{Fi}$ ) is to connect the individual microgrids to the Thing Speak server for microgrid clustering purposes.

\subsection{Internet-Based Microgrid}

Another two microgrids (MG-3 and MG-4) are also connected to the microgrid cluster. The communication medium for those microgrids is the Internet over TCP/IP. The two microgrids consist of loads only. There are no microgrid sources(MS) are present in MG-3 and MG-4 to supply the energy to the cluster. The purpose of thesetwo microgrids (MG-3 andMG-4) is tosupport the formation of a cluster of microgrids as distributed loads. These were considered in the energy management topology proposed in this work. 


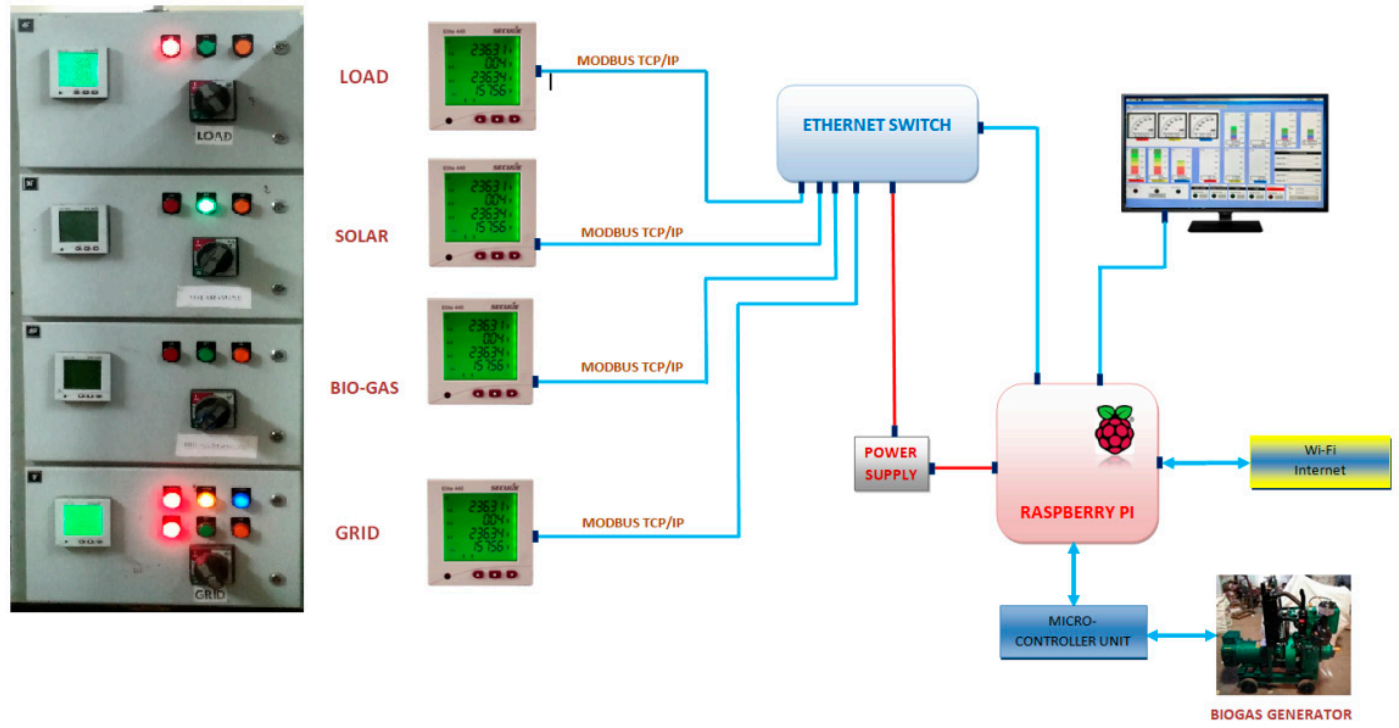

Figure 6. Schematic of Wi-fi and single-board computer-based microgrid system (Microgrid-2).

\section{Implementation of the Proposed Scheme}

\subsection{Laboratory Scale}

In the campus of IIEST, Shibpur, a lab-scale prototype of microgrid setup was established to realize the clustering between the microgrids. The site-map is shown in Figure 7. As shown in Figure 5, the microgrid-1is connected to the control and monitoring device or CMC through GSM connectivity. The data are stored in a Thing Speak-based server system where it can monitor and control the energy sources. In the microgrid lab at IIEST, Shibpur, referred to as microgrid 2, a Raspberry-Pi-based control system is used as a microgrid controller. The Raspberry Pi controller is connected to the control and monitoring device or CMC to monitor and control the sources and loads via Wi-Fi connectivity. Microgrid-3 andmicrogrid-4 are also connected to the control and monitoring device or CMC via the Internet.

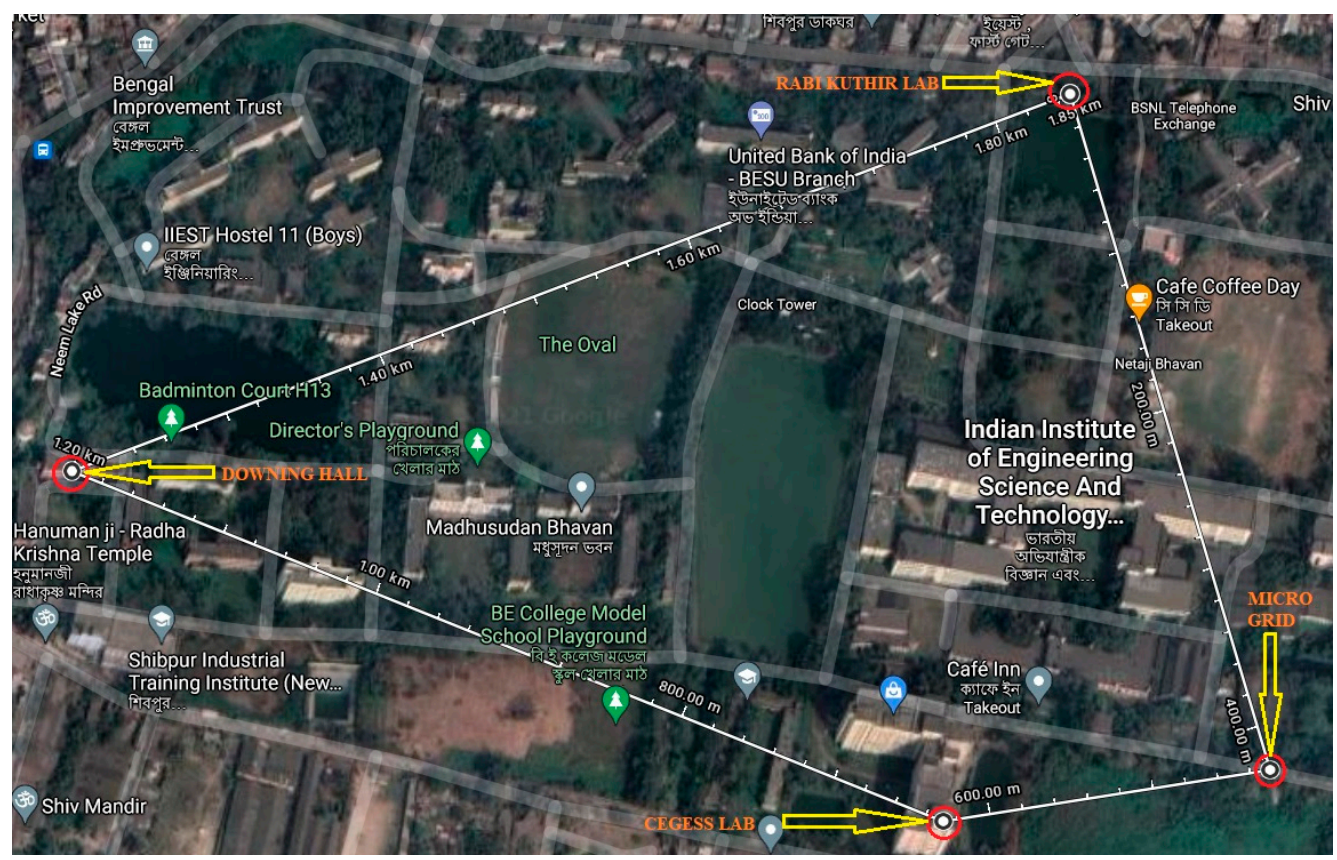

Figure 7. Google-map site view of a microgrid cluster prototype at IIEST, Shibpur. 
This kind of cluster aims to meet the energy requirement of the multiple sub-areas and ensure zero LPSP using a central control unit.

\subsection{Potential Application-Field Scale}

A rural community near Mahishmari Village at Jaynagar, West Bengal, India, was chosen to validate the proposed microgrid cluster management scheme. A survey on energy demands of two complete days was conducted. It is observed that this village consists of 240 houses. According to the input received on the energy survey, the village has a daily demand of 409 units $(409 \mathrm{kWh})$ during summer. The details are shown in Table 2. There, the people face the problem of weak distribution grid and low AC voltage issues. Hence, a microgrid cluster covering the entire area was considered as an ideal solution to the said problem. Here, the entire village is divided into three microgrid zones, as shown in Figure 8. A particular area was marked and chosen for the powerhouse of each microgrid zone, based on the density of availability of rooftops' solar PV sources and biogas power generation units. Each microgrid zone is designated by its names; “Microgrid Zone-1, Microgrid Zone-2, Microgrid Zone-3". Microgrid Zone 1 or microgrid-1 consists of $15 \mathrm{~kW}$ of a solar power plant and $15 \mathrm{~kW}$ of a biogas plant. Microgrid Zone 2 or microgrid-2 consists of $10 \mathrm{~kW}$ of a solar power plant and $20 \mathrm{~kW}$ of a biogas plant. Microgrid Zone 3 or microgrid- 3 consists of $20 \mathrm{~kW}$ of a solar power plant and $15 \mathrm{~kW}$ of a biogas plant. After forming the cluster between three microgrids, the overall solar power capacity and biogas capacity will be $45 \mathrm{kWp}$ and $50 \mathrm{kVA}$, respectively.

Table 2. Energy sources and loads of individual microgrids in the rural microgrid cluster.

\begin{tabular}{cccc}
\hline & $\begin{array}{c}\text { Solar Power Plant } \\
\text { (in kW) }\end{array}$ & $\begin{array}{c}\text { Biogas Power Plant } \\
\text { (in kVA) }\end{array}$ & $\begin{array}{c}\text { Community Load } \\
\text { (in kWh)/Day }\end{array}$ \\
\hline Microgrid-1 & 15 & 15 & 165 \\
Microgrid-2 & 10 & 20 & 118 \\
Microgrid-3 & 20 & 15 & 127 \\
\hline
\end{tabular}

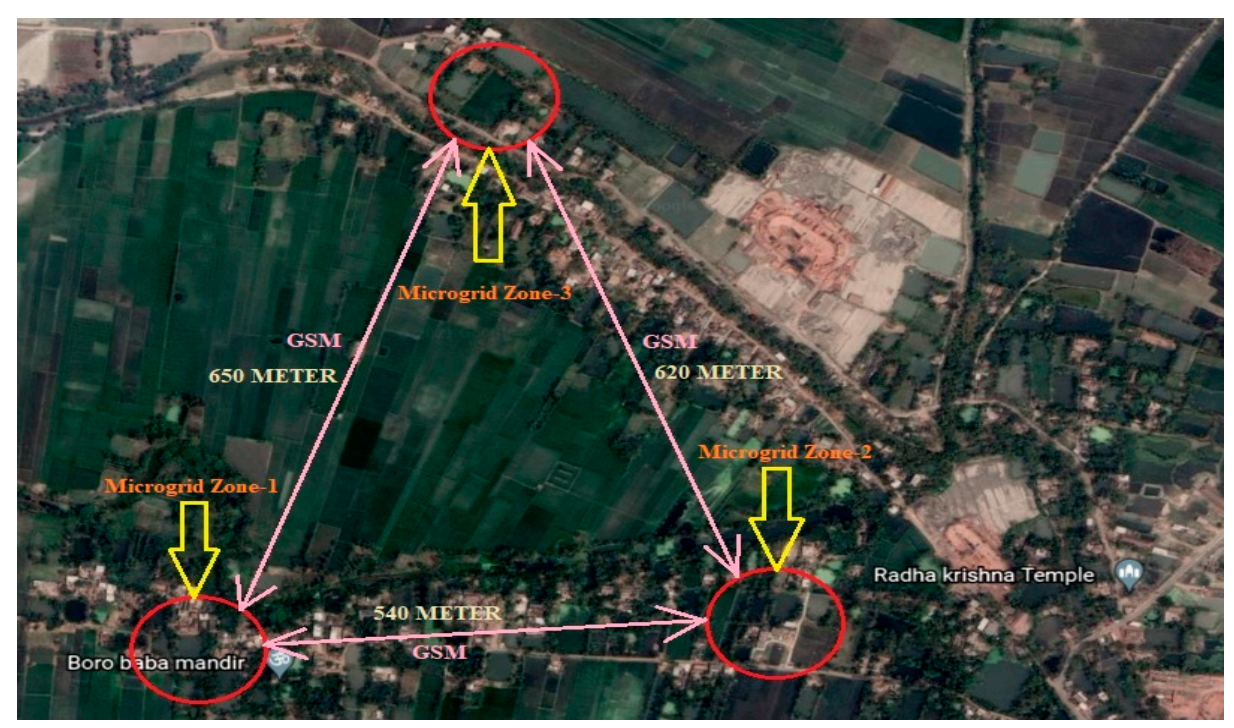

Figure 8. Google-map site view (Latitude; 22.191667 N, longitude: 88.5667 E) of the field implemented microgrid clustering. 


\subsection{Optimization of Energy Management of the Microgrid Cluster}

In order to optimize the energy management of the above-mentioned microgrid, the following Equations (1)-(4) will satisfy the objective. The variables and the objective function are defined in the given equations.

$$
\begin{aligned}
& P_{\mathrm{Mg} 1}=P_{p v 1}\left(t, I_{1}, T_{a}\right)+P_{b g 1}\left(t, Q_{1}\right) \\
& P_{\mathrm{Mg} 2}=P_{p v 2}\left(t, I_{2}, T_{a}\right)+P_{b g 2}\left(t, Q_{2}\right) \\
& P_{\mathrm{Mg} 3}=P_{p v 3}\left(t, I_{3}, T_{a}\right)+P_{b g 3}\left(t, Q_{3}\right)
\end{aligned}
$$

The power balance in the microgrid cluster is represented by Equation

$$
\sum_{\mathrm{i}=1}^{3} L_{\mathrm{i}}(\mathrm{t})=\sum P_{\mathrm{Mg} 1}(\mathrm{t}) \pm \sum P_{\mathrm{Mg} 2}(\mathrm{t}) \pm \sum P_{\mathrm{Mg} 3}(\mathrm{t}) \pm \sum P_{\mathrm{g}}(\mathrm{t})
$$

where,

$L_{\mathrm{i}}=$ Load of corresponding microgrid $(\mathrm{kW})$

$P_{b g 1}, P_{b g 2}, P_{b g 3}=$ Power generated from the biogas plant of

microgrid 1 , microgrid 2 , and microgrid 3 , respectively $(\mathrm{kW})$

$I_{1}, I_{2}, I_{3}=$ Solar insolation at three microgrid locations

$Q_{1}, Q_{2}$ and $Q_{3}=$ Gas Volume (cubic meter) for each biogas plant in the microgrids

$T_{a}=$ Ambient temperature $\left({ }^{\circ} \mathrm{C}\right)$

$P_{p v 1}, P_{p v 2}, P_{p v 3}=$ Solar power generation from microgrid 1, microgrid 2, and microgrid 3, respectively $(\mathrm{kW})$

$P_{\mathrm{g}}=$ Distribution Grid power $(\mathrm{kW})$

$P_{\mathrm{Mg} 1}, P_{\mathrm{Mg} 2}, P_{\mathrm{Mg} 3}=$ Power generation by each separate microgrid $(\mathrm{kW})$

The Figure 9 flowchart describes energy management optimization techniques for practical microgrid clusters. The flowchart proposed a GSM-based communication system to help the bidirectional communication between microgrids in a cluster. The flowchart starts with checking the GSM connection between all individual microgrids and central management controllers (CMC). The CMC is further connected to the distributed management system (DMS). In any case, if a microgrid misses the data communication with $\mathrm{CMC}$, the microgrid will be disconnected from the cluster and work as a decentralized microgrid to satisfy the local load demand. In that case, the microgrid controller is responsible for controlling the MS and LC. The CMC will control the MS and LC of the remaining microgrid present in the cluster.

In this flow chart, the power deficit of each separate microgrid is measured and compared with the threshold of power deficit of the microgrid (TPDMG). The measured data are sent to the CMC for further action. The CMC will send a command to the connection microgrid to start the alternative DERs via GSM to satisfy the energy balance of the microgrid cluster. In this case, the user can define the threshold power deficit (TPDMG) for each microgrid. The excess power generation of the microgrid cluster is exported to the grid in case of the presence of a distribution grid.

In this operation, the microgrid cluster works in a hierarchical control scheme (as shown in Figure 3d) once the GSM connection establishes with all microgrid controllers. If any microgrid is unable to communicate with the $\mathrm{CMC}$, the corresponding microgrid will be disconnected from the cluster and work in a stand-alone mode or decentralized mode of operation. This novel technique ensures the power delivery to loads of the corresponding microgrid, also avoiding the power irregularity in the cluster's bus. The remaining microgrid will be connected to the cluster control by CMC. This approach will significantly reduce the energy outage and proper utilization of RES, especially in rural areas. 


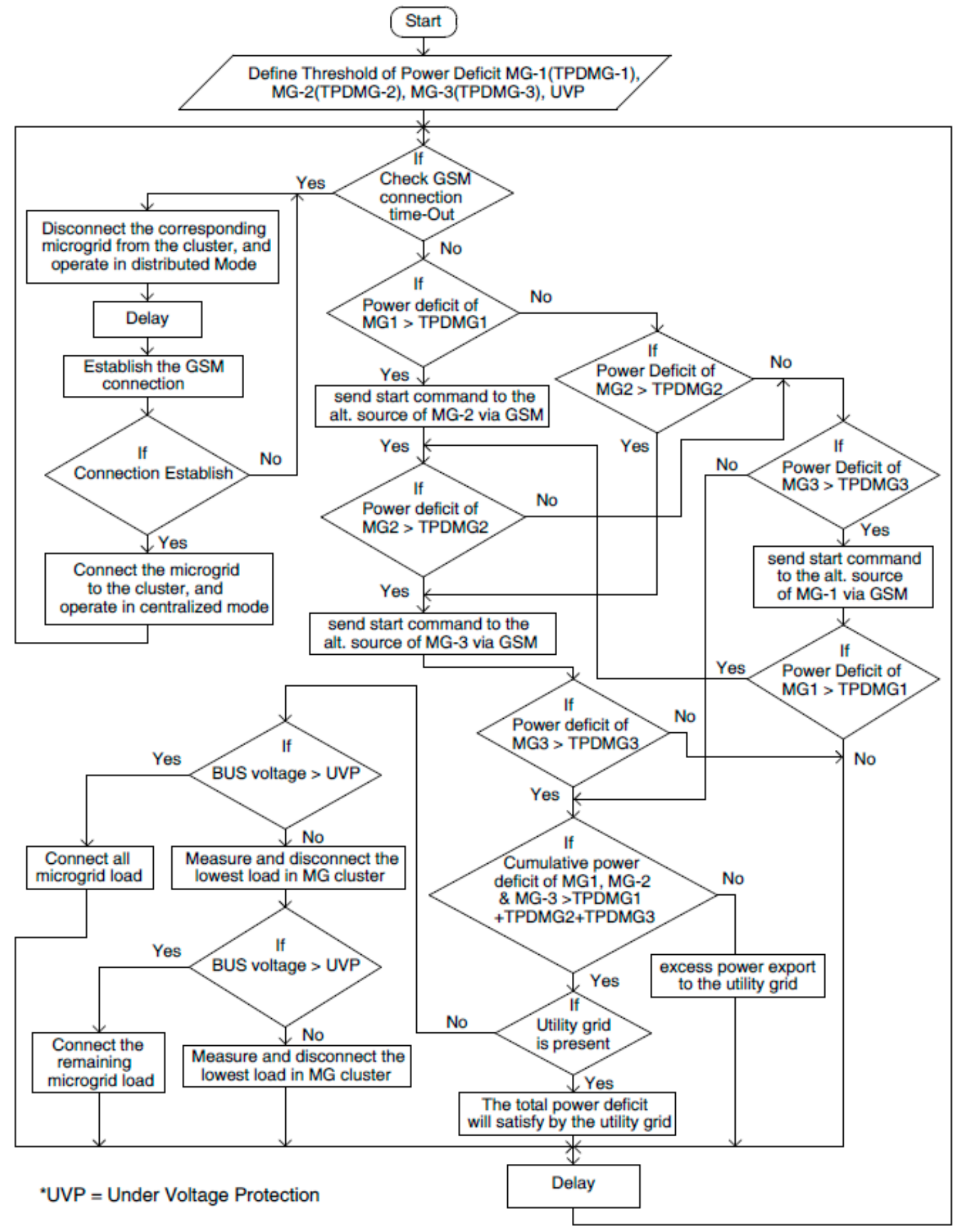

Figure 9. Flowchart representation of GSM based optimized energy management of a microgrid cluster.

\section{Result and Discussion}

As mentioned in Section 3.2, the performance of the proposed smart communication scheme and the energy management scheme was validated in the field by a practical solar PV-biogas integrated hybrid microgrid system at Mahishmari village, West Bengal, India. A complete day's practical load demand profile (6:00 AM to the next day's 5:00 AM) was chosen as the target parameter.

The peak load demand of the cluster is $28 \mathrm{~kW}$ in order to match the daily demand profile, the total solar PV of $45 \mathrm{~kW}_{\mathrm{P}}$, and the total biogas engine-generator of $50 \mathrm{kVA}$ capacity, which was integrated to form the smart hybrid microgrid. 
Figures 10-12 show three practical case studies for the demand-side management by observing the dynamics of solar generation and load profile changes in the three clusters. The solar power output profile is chosen based on the local irradiation profile throughout the day. The results in Figures 10-12 also show a special case where the uninterrupted load management was performed by the proposed energy management control strategy even during a grid outage scenario. Here, in this case, from around 6:00 PM to 7:00 PM, the grid outage occurs. Then, the biogas power generator satisfies the demand during that period as there is no solar power available in the evening.

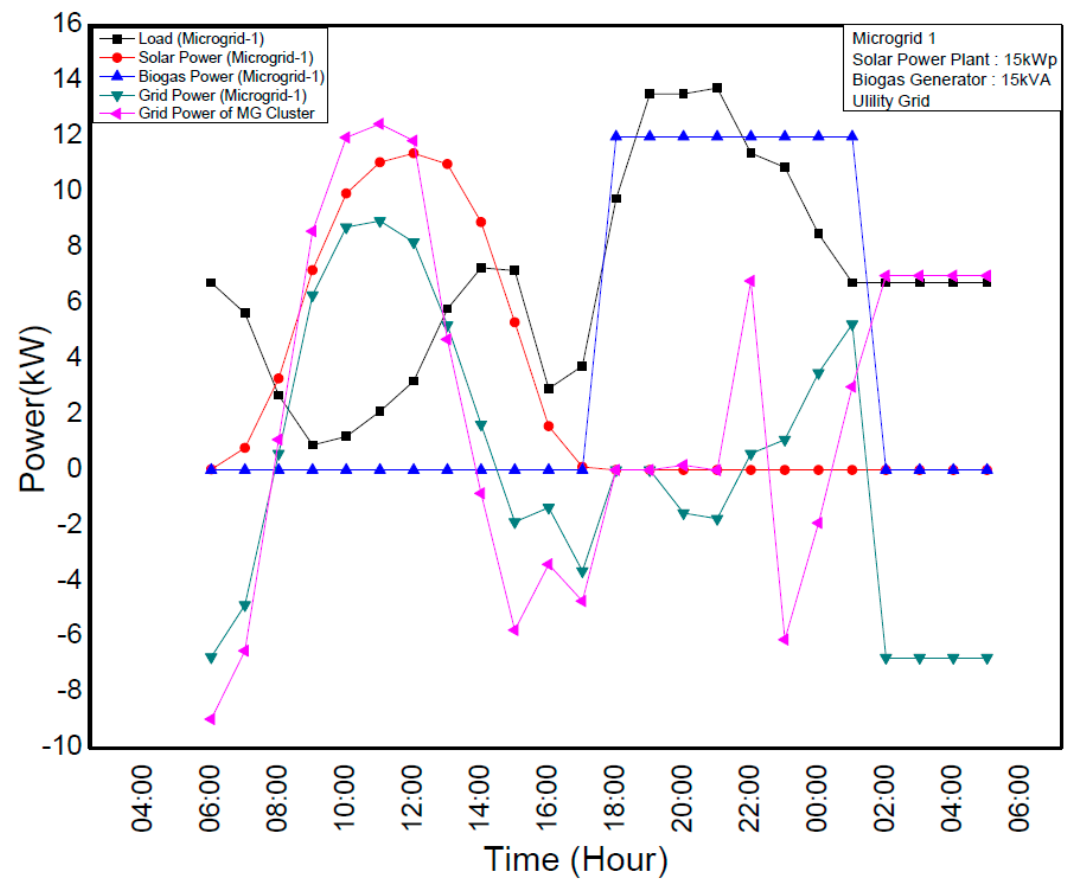

Figure 10. Demand and generation management at microgrid-1.

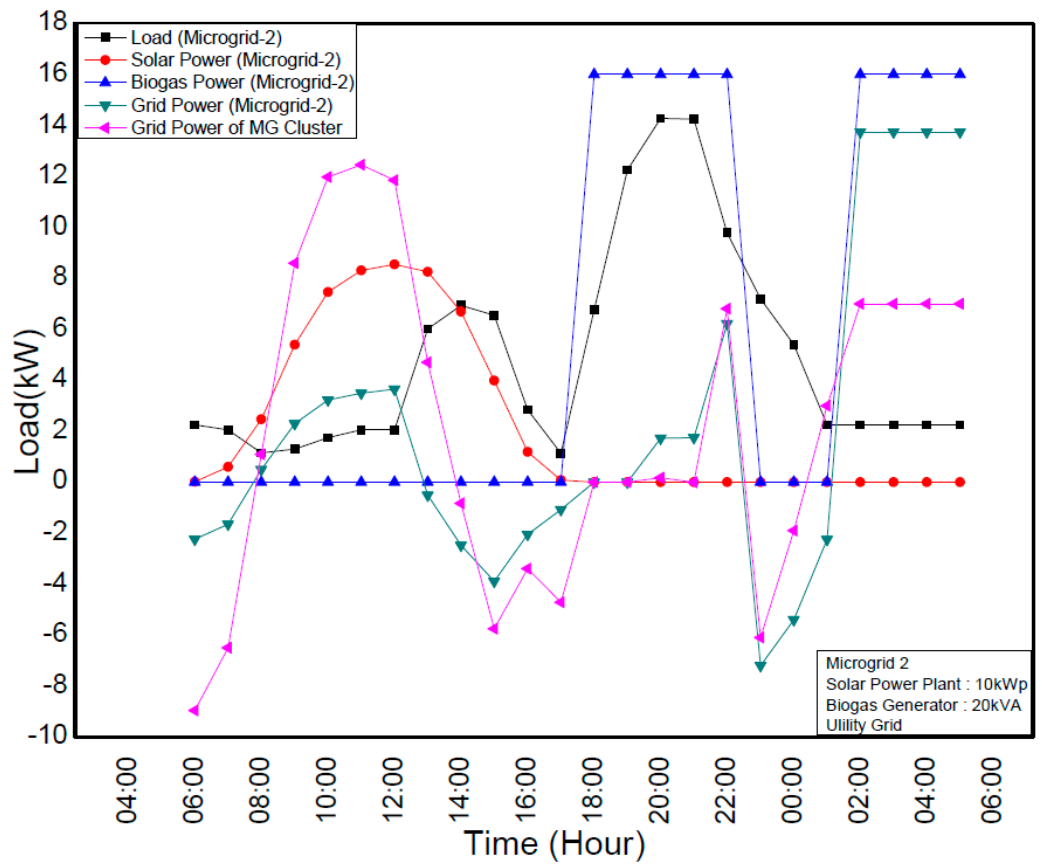

Figure 11. Demand and generation management at microgrid-2. 


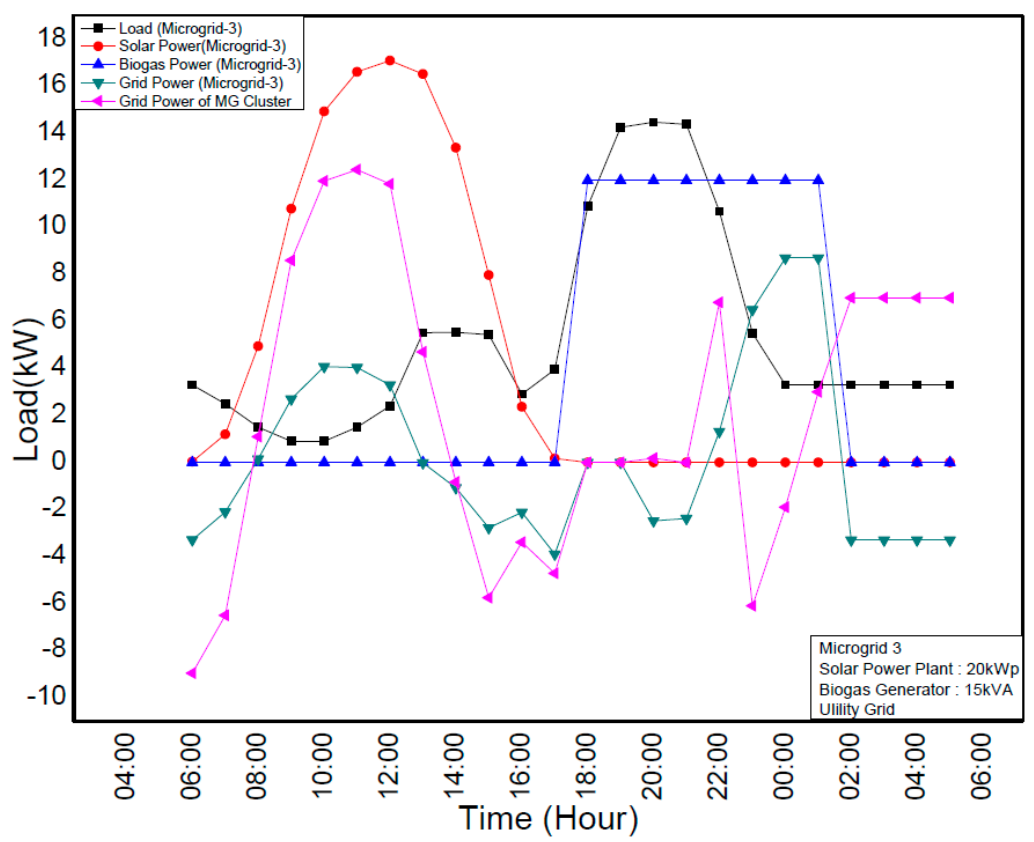

Figure 12. Demand and generation management at microgrid-3.

The total daily energy demands for each microgrid are $165 \mathrm{kWh}, 118 \mathrm{kWh}$, and $127 \mathrm{kWh}$, respectively. This daily energy demand of $409 \mathrm{kWh}$ is satisfied by optimized power-sharing among the RES (solar, biomass) and the distribution grid according to their availability.

As observed in the results, for the biogas power generator, the power never becomes negative as it acts as a reservoir and serves the load until the biogas is sufficient inside the digester. The power with a negative sign for the grid signifies the power imported from the grid during a lack of availability of RES. The grid power with a positive sign signifies power exported to the grid under excess solar PV power generation. The overall smart microgrid operation monitoring and control were executed by a communication platform that plays with the real-time data of PV power generation, biogas power generation, grid power export/import, and the connected load demand.

Figures 10-12 describe the demand and generation management within the three microgrid clusters. The red line represents the solar generation by three microgrids. The blue line describes the biogas electricity generation by three microgrids. The black line describes the load demand by the microgrids. The green line describes energy supplied by the distribution grid to satisfy demand beyond the individual generation of each microgrid. Finally, the pink line represents the overall consumption from the distribution grid by the three microgrid cluster system.

\section{Conclusions}

In this paper, a GSM-based smart communication-based energy management scheme is developed and validated in the field by a practical solar PV and biogas plant integrated clustered microgrid system. For preliminary demonstration and testing of the cluster system on a laboratory scale, a prototype of the same system consisting of integrated solar PV and biogas plant was developed at IIEST, Shibpur campus. An optimized energy management scheme was developed and demonstrated in a field application of a village containing multiple microgrid clusters.

The field experiment results demonstrate that the dynamic load demand profiles of the chosen interconnected microgrid areas are satisfied by the optimized scheduling of RES, and the grids. These components have been well utilized in the real-time control and exchange of power between the connected microgrids. The smart energy management 
scheme developed in this work is a generalized one and can be scaled up for any multicluster community energy management.

Author Contributions: Conceptualization, Methodology, and Writing-original draft preparation, (H.S. (Hiranmay Samanta), A.B. and J.J.), Software (A.D.), Validation (I.B.), Writing-review and editing, and supervision (H.S. (Hiranmay Saha), K.D.B. and S.S.). All authors have read and agreed to the published version of the manuscript.

Funding: This research is funded by U.K. India Clean Energy Research Institute under Grant DST/RCUK/JVCCE/2015/02(C).

Institutional Review Board Statement: Not applicable.

Informed Consent Statement: The research was carried out at the School of Advanced Materials, Green Energy and Sensor Systems, IIEST, Shibpur, India. The first author Hiranmay Samanta would like to acknowledge the fellowship support given by Department of Science and Technology (DST), Government of India (GoI), to carry out the research. This work was conducted as part of the research project 'Joint UK-India Clean Energy Centre (JUICE)', which is funded by the RCUK's Energy Programme (contract no: EP/P003605/1). The project funders were not directly involved in the writing of this article. In support of open access research, all underlying article materials (data, models) can be accessed upon request via email to the corresponding author.

Data Availability Statement: Not applicable.

Acknowledgments: This work has been supported by the UK-India Clean Energy Research Institute.

Conflicts of Interest: The authors declare that there is no conflict of interest regarding the publication of this paper.

\section{References}

1. Kumar, A.; Singh, A.R.; Deng, Y.; He, X.; Kumar, P.; Bansal, R.C. Integrated assessment of a sustainable microgrid for a remote village in hilly region. Energy Convers. Manag. 2019, 180, 442-472. [CrossRef]

2. Samanta, H.; Bhattacharjee, A.; Pramanik, M.; Das, A.; Das Bhattacharya, K.; Saha, H. Internet of things based smart energy management in a vanadium redox flow battery storage integrated bio-solar microgrid. J. Energy Storage 2020, $32,101967$. [CrossRef]

3. Khan, M.R.B.; Jidin, R.; Pasupuleti, J. Multi-agent based distributed control architecture for microgrid energy management and optimization. Energy Convers. Manag. 2016, 112, 288-307. [CrossRef]

4. Li, Y.; Nejabatkhah, F. Overview of control, integration and energy management of microgrids. J. Mod. Power Syst. Clean Energy 2014, 2, 212-222. [CrossRef]

5. Seifi, A.; Moradi, M.H.; Abedini, M.; Jahangiri, A. An optimal programming among renewable energy resources and storage devices for responsive load integration in residential applications using hybrid of grey wolf and shark smell algorithms. J. Energy Storage 2020, 27, 101126. [CrossRef]

6. Kumar, K.P.; Saravanan, B. Day ahead scheduling of generation and storage in a microgrid considering demand Side management. J. Energy Storage 2019, 21, 78-86. [CrossRef]

7. Zia, M.F.; Elbouchikhi, E.; Benbouzid, M. Microgrids energy management systems: A critical review on methods, solutions, and prospects. Appl. Energy 2018, 222, 1033-1055. [CrossRef]

8. Sarkar, T.; Bhattacharjee, A.; Samanta, H.; Bhattacharya, K.; Saha, H. Optimal design and implementation of solar PV-windbiogas-VRFB storage integrated smart hybrid microgrid for ensuring zero loss of power supply probability. Energy Convers. Manag. 2019, 191, 102-118. [CrossRef]

9. Hosseini, S.J.A.D.; Moazzami, M.; Shahinzadeh, H. Optimal sizing of an isolated hybrid wind/PV/battery system with considering loss of power supply probability. Majlesi J. Electr. Eng. 2017, 11, 63-69.

10. Abbes, D.; Martinez, A.; Champenois, G. Life cycle cost, embodied energy and loss of power supply probability for the optimal design of hybrid power systems. Math. Comput. Simul. 2014, 98, 46-62. [CrossRef]

11. Liu, Z.; Yang, J.; Jiang, W.; Wei, C.; Zhang, P.; Xu, J. Research on Optimized Energy Scheduling of Rural Microgrid. Appl. Sci. 2019, 9, 4641. [CrossRef]

12. Gaona, E.E.; Trujillo, C.L.; Guacaneme, J.A. Rural microgrids and its potential application in Colombia. Renew. Sustain. Energy Rev. 2015, 51, 125-137. [CrossRef]

13. Marín, L.G.; Sumner, M.; Muñoz-Carpintero, D.; Köbrich, D.; Pholboon, S.; Sáez, D.; Núñez, A. Hierarchical energy management system for microgrid operation based on robust model predictive control. Energies 2019, 12, 4453. [CrossRef]

14. Bullich-Massagué, E.; Díaz-González, F.; Aragüés-Peñalba, M.; Girbau-Llistuella, F.; Olivella-Rosell, P.; Sumper, A. Microgrid clustering architectures. Appl. Energy 2018, 212, 340-361. [CrossRef] 
15. Bandeiras, F.; Pinheiro, E.; Gomes, M.; Coelho, P.; Fernandes, J. Review of the cooperation and operation of microgrid clusters. Renew. Sustain. Energy Rev. 2020, 133, 110311. [CrossRef]

16. Rostami, Z.; Ravadanegh, S.N.; Kalantari, N.T.; Guerrero, J.M.; Vasquez, J.C. Dynamic Modeling of Multiple Microgrid Clusters Using Regional Demand Response Programs. Energies 2020, 13, 4050. [CrossRef]

17. Yigit, M.; Gungor, V.C.; Baktir, S. Cloud Computing for Smart Grid applications. Comput. Netw. 2014, 70, 312-329. [CrossRef]

18. Aghenta, L.O.; Iqbal, M.T. Design and implementation of a low-cost, open source IoT-based SCADA system using ESP32 with OLED, ThingsBoard and MQTT protocol. AIMS Electron. Electr. Eng. 2019, 4, 57-86. [CrossRef]

19. Wu, Y.; Wu, Y.; Guerrero, J.M.; Vasquez, J.C.; Palacios-García, E.J.; Guan, Y. IoT-enabled microgrid for intelligent energy-aware buildings: A novel hierarchical self-consumption scheme with renewables. Electronics 2020, 9, 550. [CrossRef]

20. Majee, A.; Bhatia, M.; Swathika, O.V.G. IoT Based Microgrid Automation for Optimizing Energy Usage and Controllability. In Proceedings of the 2018 Second International Conference on Electronics, Communication and Aerospace Technology (ICECA), Coimbatore, India, 29-31 March 2018; pp. 685-689.

21. Nandipati, S.H.; Babu, P.T.; Chigurupati, M.; Vaithilingam, C. Interface Protection and Energy Management System for Microgrid using Internet of Things. Energy Procedia 2017, 117, 201-208. [CrossRef]

22. Prakosa, S.W.; Faisal, M.; Adhitya, Y.; Leu, J.-S.; Köppen, M.; Avian, C. Design and Implementation of LoRa Based IoT Scheme for Indonesian Rural Area. Electronics 2021, 10, 77. [CrossRef]

23. Ndukwe, C.; Iqbal, M.T.; Liang, X.; Khan, J.; Aghenta, L. LoRa-based communication system for data transfer in microgrids. AIMS Electron. Electr. Eng. 2020, 4, 303-325. [CrossRef]

24. Kou, P.; Liang, D.; Gao, L. Distributed EMPC of multiple microgrids for coordinated stochastic energy management. Appl. Energy 2017, 185, 939-952. [CrossRef]

25. Mao, M.; Wang, Y.; Chang, L.; Du, Y. Operation optimization for multi-microgrids based on centralized-decentralized hybrid hierarchical energy management. In Proceedings of the 2017 IEEE Energy Conversion Congress and Exposition (ECCE), Cincinnati, OH, USA, 1-5 October 2017; pp. 4813-4820.

26. Wang, P.; Zhang, Y.; Yang, H. Research on Economic Optimization of Microgrid Cluster Based on Chaos Sparrow Search Algorithm. Comput. Intell. Neurosci. 2021, 1-18. [CrossRef]

27. Kayem, A.V.D.M.; Meinel, C.; Wolthusen, S.D. A Resilient Smart Micro-Grid Architecture for Resource Constrained Environments. Adv. Inf. Secur. 2018, 71, 71-101.

28. Zhao, X.; Liu, S. Design of a Monitoring System of Micro-Grid. Smart Grid Renew. Energy 2013, 4, 198-201. [CrossRef]

29. Pulcherio, M.; Illindala, M.S.; Choi, J.; Yedavalli, R.K. Robust Microgrid Clustering in a Distribution System with Inverter-Based DERs. IEEE Trans. Ind. Appl. 2018, 54, 5152-5162. [CrossRef] 